\title{
Spatially resolved modelling of inhomogeneous materials with a first order magnetic phase transition
}

Nielsen, Kaspar Kirstein; Bahl, Christian; Smith, Anders; Bjørk, Rasmus

Published in:

Journal of Physics D: Applied Physics

Link to article, DOI:

10.1088/1361-6463/aa86e2

Publication date:

2017

Document Version

Peer reviewed version

Link back to DTU Orbit

Citation (APA):

Nielsen, K. K., Bahl, C., Smith, A., \& Bjørk, R. (2017). Spatially resolved modelling of inhomogeneous materials with a first order magnetic phase transition. Journal of Physics D: Applied Physics, 50(41), [414002].

https://doi.org/10.1088/1361-6463/aa86e2

\section{General rights}

Copyright and moral rights for the publications made accessible in the public portal are retained by the authors and/or other copyright owners and it is a condition of accessing publications that users recognise and abide by the legal requirements associated with these rights.

- Users may download and print one copy of any publication from the public portal for the purpose of private study or research

- You may not further distribute the material or use it for any profit-making activity or commercial gain

- You may freely distribute the URL identifying the publication in the public portal 


\title{
Spatially resolved modelling of inhomogeneous materials with a first order magnetic phase transition
}

\author{
K.K. Nielsen, C.R.H. Bahl, A. Smith, R. Bjørk \\ Technical University of Denmark, Frederiksborgvej 399, 4000 Roskilde, Denmark \\ E-mail: kaki@dtu.dk
}

\begin{abstract}
We present a numerical model that can simulate a magnetocaloric sample on the grain size level, including magnetostatics, heat transfer, local hysteresis and spatial variation of stoichiometry expressed as a variation in Curie temperature, $T_{\mathrm{C}, 0}$. Grain structure of a sample is realized as a number of regions each having a uniform $T_{\mathrm{C}, 0}$ and defined through a Voronoi-map. We show that demagnetising effects, caused by a finite sample size, and spatial variation in $T_{\mathrm{C}, 0}$ can account for the previously experimentally observed "virgin" effects in the adiabatic temperature change and isothermal entropy change, respectively and first order reversal effect as a function of temperature. We conclude that even a very little variation in local stoichiometry of less than a percent, corresponding to a standard deviation in $T_{\mathrm{C}, 0}$ of $\sigma_{T_{\mathrm{C}, 0}}=2 \mathrm{~K}$ for $\mathrm{La}(\mathrm{Fe}, \mathrm{Si}, \mathrm{Mn}){ }_{13} \mathrm{H}$ has a significant impact on the overall properties and history dependence of a sample.
\end{abstract}

PACS numbers: 75.30.Sg, 75.60.-d, 75.50.Cc,75.30.Kz,75.80.+q

Keywords: First order phase change, Magnetocaloric, Hysteresis, model Submitted to:

J. Phys. D: Appl. Phys. 


\section{Introduction}

A number of magnetic materials have a structural phase transition at a temperature below their magnetic transition, resulting in a discontinuity in the magnetisation. This is known as a first order magnetic phase transition (FOPT). Associated with such a phase transition is often a thermal hysteresis, making the exact state of a sample history dependent in the mixed-phase region. Due to the field dependence of the transition temperatures, a magnetic hysteresis is also present, and indeed the FOPT materials are characterised as having thermal and magnetic hysteresis. It should be noted however that the magnetic hysteresis differs from the conventional anisotropy driven type found in hard ferromagnets, in that there is no remanent magnetisation or coercivity [1].

The discontinuous loss of magnetic order resulting in a large isothermal entropy change, $\Delta S_{\mathrm{m}}$, in FOPT materials makes them of special interest for the field of magnetocalorics and the derived technology of magnetic refrigeration [1]. In the past decade new magnetocaloric materials have been developed, many of these with a FOPT, and magnetocaloric demonstration devices have become ever more powerful and efficient [2]. Any utilisation of the materials in such devices relies on the detailed understanding of these and to some extent control of their properties.

Experimentally the region around the FOPT can be probed by careful measurement using a suitably designed procedure [1, 3]. Such a procedure will often rely on the thermal resetting of the sample between each measurement in order to map out the hysteresis region[4]. It has been show that failure to do this resetting will result in lower values of the measured properties, such as the isothermal entropy change [5]. However, it has also been shown how these lower properties are actually the ones experienced in magnetocaloric devices due to the cyclic nature of their operation [6].

Another magnetic characterisation method which is gaining in popularity is to use first order reversal curve (FORC) analysis [7, 8]. A FORC is constructed by measuring minor loops in the hysteresis region.

Modelling of the FOPT materials has largely relied on the use of Preisach type schemes $[9,10,11]$. These models rely on the input of experimental data, often from FORC analysis, to keep track of the hysteresis region and predict the properties of the materials. While the Preisach schemes have proven to be powerful tools in the analysis of FOPT materials, the direct physical meaning of the input parameters is not always clear. Here we present a model framework where the only physical input is the magnetic state function of the material, which is then used to calculate the magnetic and magnetocaloric properties, as functions of the temperature and applied magnetic field.

Real samples are generally not completely homogeneous. Local chemical variations or other imperfections in the sample may result in a spatial variation of transition temperatures, which due to the sharp nature of the transition become more pronounced in materials with a FOPT. Experimental characterisation of such inhomogeneous samples suffers from the fact the most measurements are bulk measurements giving an average value of, e.g., the magnetisation in a certain volume. Only few methods allow for analysis at a level where the sample can be considered to be locally homogeneous. One example of spatially resolving the inhomogeneity in samples is demonstrated in Ref. [12]. Here a scanning 
Hall probe is used to show how the phase transition starts as local nucleation and evolves across the sample. In an inhomogeneous sample the magnetic state can be very complicated as each area has its own local hysteretic properties and history. In addition, each area will interact magnetically with its surroundings, changing the local magnetic field and thus transition temperatures around it. Applying a certain magnetic field to the sample will thus result in a distribution of internal fields depending on the shape of the sample and distribution of magnetisation within it. Overall demagnetisation corrections due to the shape of the sample are well known, see, e.g. [13], but for inhomogeneous FOPT materials it is critically important that the magnetic field is finely resolved throughout the sample, as the sharp nature of the transition and the hysteresis may result in significant local variations, which can change the overall behavior of the sample.

Based on the state function of the material, the shape of the sample and a map of the transition temperature distribution the model presented in the following can calculate the magnetic and magnetocaloric properties both locally and globally for a spatially inhomogeneous sample. When applying and removing a magnetic field to study the magnetocaloric properties the history of the sample becomes important. So whenever the magnetic response to a change in magnetic field or temperature is calculated any previous history is taken into account.

We here choose model parameters that reflect the values of the materials series $\mathrm{La}\left(\mathrm{Fe}_{x} \mathrm{Mn}_{y} \mathrm{Si}_{z}\right){ }_{13} \mathrm{H}_{1.65}$ where $x+y+z=1$. It is not, however, the intent of the present paper to accurately model a specific sample or materials series, but rather to investigate some of the resulting magnetic properties in magnetocaloric compounds with a spatial variation of stoichiometry and thus Curie temperature and the variations of the local field in a sample. In the following, a Gaussian distribution is assumed for the spread in Curie temperature and a fixed standard deviation of $\sigma_{T_{\mathrm{C}, 0}}=2 \mathrm{~K}$ is assumed. Considering the results presented in [14], a variation of $2 \mathrm{~K}$ in $T_{\mathrm{C}, 0}$ corresponds to an absolute variation of 0.0115 in the Mn contents ( $y$ in the chemical formula). With $T_{\mathrm{C}, 0}$ around room temperature the value of $y$ is around 0.30 [14], i.e. a relative variation of $\mathrm{Mn}$ content of $3 \%$ results in a variation of $2 \mathrm{~K}$ in Curie temperature.

Other dynamical effects influencing the bulk magnetocaloric properties include local stresses due to the volume change across the phase transition. In an inhomogeneous sample the sample is expected to cause stress on itself by these local volume changes and this will definitely influence the overall properties. Furthermore, exchange coupling at the interface between grains is expected to play a significant role as well. These two effects are not taken into account in the present paper but will be the subjects of future publications.

\section{Model}

The model consists of a number, $n$, of rectangular prisms each having individual geometrical properties, i.e. location, $\mathbf{r}=$ $(x, y, z)$ and dimensions, $D=(a, b, c)$. Each prism also has an individual magnetic state function, here characterized by a Curie temperature, $T_{\mathrm{C}, 0}$, and the current state (para- or ferro-magnetic) defined through a binary parameter, $h$, with $h=0$ denoting the paramagnetic state and $h=1$ the ferromagnetic state. 


\subsection{Magnetostatics}

The local magnetic field, $\mathbf{H}_{i}$, of the $i$ 'th prism is found by assuming the magnetisation of the $i$ 'th prism to be constant throughout the prism and parallel to the local field. This assumes that the material is a soft ferromagnet. The field produced by prism $i$ with magnetisation $\mathbf{M}_{i}$ at the location $\mathbf{r}$ is given by:

$\mathbf{H}(\mathbf{r})=-\mathbb{N}\left(\mathbf{r}-\mathbf{r}_{i}, D_{i}\right) \cdot \mathbf{M}_{i}\left(\mathbf{H}\left(\mathbf{r}_{i}\right), \mathbf{r}_{i}, T_{i}\right)$,

where $\mathbb{N}$ is the demagnetisation tensor field, which is a function of the relative position of the $i$ th cell, the point at which the field is evaluated and the dimensions of the $i$ 'th cell, $D_{i}$. The components of the demagnetisation tensor are given in Appendix $A$ and the derivation may be found in [15]. The magnetisation, $\mathbf{M}$, depends on the temperature, $T$, as well as the magnetic field.

In total, the local field at the $i$ 'th prism is given by the super position of the fields from all the other prisms and the applied field, $\mathbf{H}_{\mathrm{app}}$ :

$$
\begin{aligned}
& \mathbf{H}_{i}\left(\mathbf{r}_{i}\right)=\mathbf{H}_{\mathrm{app}}\left(\mathbf{r}_{i}\right) \\
& -\sum_{j=1, j \neq i}^{j=n} \mathbb{N}\left(\mathbf{r}_{i}-\mathbf{r}_{j}, D_{j}\right) \cdot \mathbf{M}_{j}\left(\mathbf{H}\left(\mathbf{r}_{j}\right), \mathbf{r}_{j}, T_{j}\right) .
\end{aligned}
$$

Since the solution depends on the local magnetisation in each prism, which in turn depends on the local field and temperature, Eq. 2 is solved by iteration.

\subsection{Magnetisation and entropy}

Each prism is assumed to have an independent state function, i.e. the magnetisation and entropy as a function of magnetic field and temperature are specified for each prism individually. In the present paper, the state function proposed by Bean and Rodbell [16] is assumed. This model has been applied with some degree of success in explaining experimental results of first order phase transitions [17, 18], which is in part attributed to the fact that spin fluctuations are less pronounced in a FOPT material as the transition is well below the purely magnetic transition, which would be the transition temperature in the absence of a structural change.

The Bean-Rodbell model is a mean field model which in addition assumes a linear relationship between the exchange constant, $\lambda$ and the volume, $\frac{V-V_{0}}{V_{0}}$ :

$\lambda=\lambda_{0}\left(1+\beta \frac{V-V_{0}}{V_{0}}\right)$,

which in the mean field model results in

$T_{\mathrm{C}}=T_{\mathrm{C}, 0}\left(1+\beta \frac{V-V_{0}}{V_{0}}\right)$,

where $V$ is the unit cell volume and $\lambda_{0}, V_{0}$ and $T_{\mathrm{C}, 0}$ are the exchange constant, the unit cell volume and Curie temperature in the case with no magneto-elastic coupling, respectively. The parameter, $\beta$, describes the strength of the magneto-elastic coupling and is typically expressed in terms of the so-called BeanRodbell parameter, $\eta$ :

$\eta=40 N k_{\mathrm{B}} T_{\mathrm{C}, 0} \kappa \beta^{2} \frac{(J(J+1))^{2}}{(2 J+1)^{4}-1}$,

with $N$ denoting the number of magnetic ions per unit volume, $J$ being their total angular momentum, $\kappa$ the compressibility and $k_{\mathrm{B}}$ is Boltzmann's constant. When $0 \leq \eta \leq 1$ the transition is second order, and when $\eta>1$ the transition is first order. The free energy is given by:

$F(T, H, p)=-T S_{\mathrm{mag}}-M H$

$-\frac{1}{2} \lambda M^{2}+\frac{1}{2 \kappa}\left(\frac{V-V_{0}}{V_{0}}\right)^{2}+p \frac{V-V_{0}}{V_{0}}$.

Here, the magnetic entropy is denoted $S_{\text {mag }}$ and the absolute pressure is $p$. In the present study $p$ is assumed zero throughout. It is noted that the norm of the local 
field, $H=\sqrt{H_{x}^{2}+H_{y}^{2}+H_{z}^{2}}$, and the norm of the magnetisation, $M$, are used in the free energy. Equation 6 is minimized with respect to volume and subsequently with respect to magnetisation. The first step is done analytically resulting in the equilibrium volume:

$\frac{V-V_{0}}{V_{0}} \mid=\frac{1}{2} \lambda_{0} \beta \kappa M^{2}-p \kappa$.

The magnetic entropy is

$S_{\mathrm{mag}}=k_{\mathrm{B}} N\left(\ln \frac{\sinh \left(\frac{2 J+1}{2 J} x\right)}{\sinh \left(\frac{1}{2 J} x\right)}-x B_{J}(x)\right)$,

with the Brillouin function

$$
\begin{aligned}
B_{J}(x) & =\frac{2 J+1}{2 J} \operatorname{coth}\left(\frac{2 J+1}{J} x\right) \\
& -\frac{1}{2 J} \operatorname{coth}\left(\frac{1}{2 J} x\right),
\end{aligned}
$$

$x=\frac{\gamma J}{k_{\mathrm{B}} T}(H+\lambda M)$ and $\gamma=\mu_{\mathrm{B}} g$ where $\mu_{\mathrm{B}}$ and $g=2$ are the Bohr magneton and the Landé factor, respectively.

The above expression for $S_{\text {mag }}$ along with the equilibrium volume (Eq. 7) is inserted into the free energy (Eq. 6):

$F(T, H, p)=-k_{\mathrm{B}} T N \ln \frac{\sinh \left(\frac{2 J+1}{2 J} x\right)}{\sinh \left(\frac{1}{2 J} x\right)}$

$+\frac{1}{2} \lambda_{0} M^{2}(1-\beta \kappa p)$

$+\frac{3}{8} \lambda_{0}^{2} M^{4} \beta^{2} \kappa-\frac{1}{2} \kappa p^{2}$.

Equation 10 should be minimized with respect to magnetisation, i.e. finding the roots of

$$
\begin{aligned}
\frac{\partial F}{\partial M}= & -\gamma \lambda_{0} J N B_{J}(x)\left(1+\frac{3}{2} \lambda_{0} \beta^{2} \kappa M^{2}-\beta \kappa p\right) \\
& +\lambda_{0} M(1-\beta \kappa p)+\frac{3}{2} \lambda_{0}^{2} M^{3} \beta^{2} \kappa=0 .
\end{aligned}
$$

This is done numerically. The free energy may have two minima in $M$; a stable and a metastable minimum. The stable minimum corresponds to the magnetisation found while cooling a sample from a high temperature

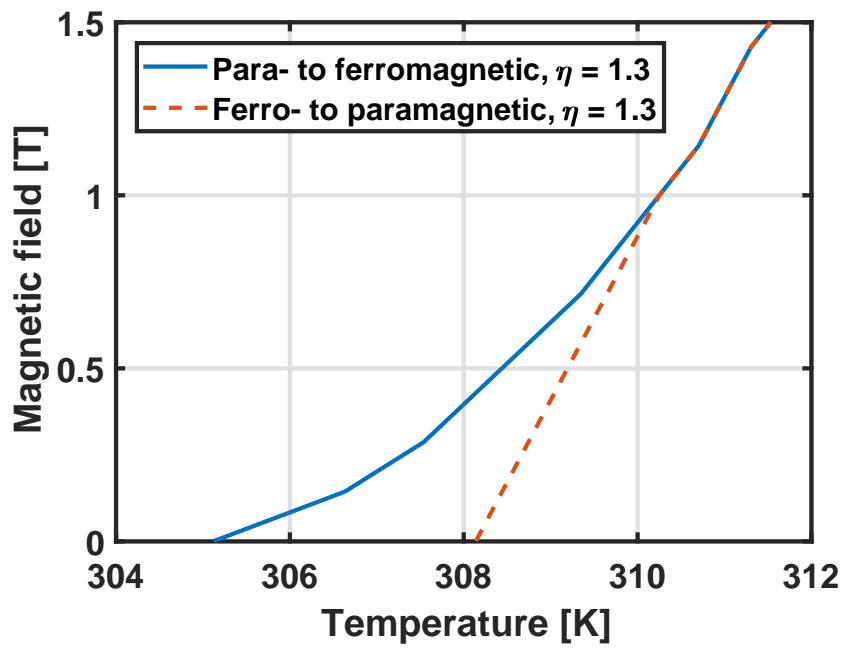

Figure 1. Phase diagram for a single prism obtained from minimizing the free energy (Eq. 6) with respect to volume and magnetisation with $\eta=1.3$ and parameters as given in Table 1 . The prism is ferromagnetic when the state is above the blue (full) line and paramagnetic when below the red (dashed) line. The state in between the two lines is determined from the history. The pressure $p$ is zero.

while the metastable minimum corresponds to the magnetisation found when heating the sample from a low temperature. This effect gives the thermal and magnetic hysteresis directly and is only present when $\eta>1$. Once the magnetisation has been found as a function of temperature, field and pressure the magnetic entropy may be found using Eq. 8.

Figure 1 shows the phase diagram of a single prism computed using the parameters given in Table 1. The two phase lines are separated from $H=0 \mathrm{~T}$ to $H=0.8 \mathrm{~T}$. When the state, i.e. the temperature and field of the prism, is above the blue (full) line, the prism is ferromagnetic. When the state is below the red (dashed) line the prism is paramagnetic. In the region separating the two lines the state of the prisms is given by its history, i.e. a prism being heated from a low temperature will stay ferromagnetic until reaching the red (dashed) line, while a prism 
being cooled from a high temperature will stay paramagnetic until reaching the blue (solid) line.

The choice of the parameters in Table 1 are chosen to mimic the parameters of $\mathrm{La}(\mathrm{Fe}, \mathrm{Mn}, \mathrm{Si}){ }_{13} \mathrm{H}$, not to model this material in detail. This choice of parameters is deliberately not tuned to model a specific experimental data set, but rather to be instructive and still highly relevant.

The lattice and electronic contributions to the entropy are assumed to follow the Debye and Sommerfeld models, respectively. These are given by:

$$
\begin{aligned}
S_{\text {lat }} & =-3 N_{\text {atom }} k_{\mathrm{B}}\left(\ln \left(1-e^{-\frac{T_{\mathrm{D}}}{T}}\right)\right) \\
& -4 D\left(\frac{T_{\mathrm{D}}}{T}\right) \\
D(x) & =\frac{1}{x^{3}} \int_{0}^{x} \frac{y^{3}}{e^{y}-1} \mathrm{~d} y . \\
S_{\text {ele }} & =\gamma_{e} T,
\end{aligned}
$$

with the Sommerfeld constant $\gamma_{e}$. Here $N_{\text {atom }}$ denotes the total number of atoms per unit volume and $T_{\mathrm{D}}$ is the Debye temperature. The values in Table 1 are used throughout this work, and are selected to match those of $\mathrm{La}(\mathrm{Fe}, \mathrm{Mn}, \mathrm{Si})_{13} \mathrm{H}$.

\subsection{Sample generation and meshing}

The lattice and electronic entropy could have different temperature dependencies in the two phases and this is not currently taken into account in the model.

There are no specific requirements for the geometry of the modelled sample. In the present case we consider flat samples and employ a sample generation strategy that i) makes quadratic domains and ii) introduces chemical heterogeneity using Voronoi-maps [20]. Given an initial resolution in the $x-$ and $y$-dimensions, i.e. a number of prisms $n_{x}$ and $n_{y}$ in either direction, the modelled domain is generated through a distribution of $\lambda_{0}$, or equivalently $T_{\mathrm{C}, 0}$, specified by the number of different values $n_{T_{\mathrm{C}, 0}}$ and any distribution parameters, i.e. the standard deviation, $\sigma_{T_{\mathrm{C}, 0}}$ in the case of a Gaussian distribution. The locations of the $n_{T_{\mathrm{C}, 0}}$ points are chosen randomly within the bounds of the sample and subsequently a Voronoi-map is created based on this distribution. In short, a Voronoimap consists of a number of homogeneous regions. The borders between these regions are defined as having equal distance to two or more specified points; in this case the randomly specified points as described above. As can be seen in Fig. 2(c) this results in a grain-like structure and each region is assumed to model a single grain.

The meshing starts with a uniform grid with a specified resolution $\left(n_{x}\right.$ and $\left.n_{y}\right)$ that is refined $L$ times. In each refinement step each prism, called a parent, is split into four equally sized smaller prisms, called children. Once the maximum refined mesh has been generated, the child prisms of each individual parent are investigated, by checking whether the children are closer to another of the $n_{T_{\mathrm{C}, 0}}$ random points than the parent prism is. If this is the case the children remain. Otherwise, the children are destroyed and the parent prism remains. Figure 2 shows an example of a Voronoi-map generated and meshed with this strategy. In total there are 5514 prisms in Figure 2. As can clearly be seen from the figure, this way of meshing introduces a detailed mesh on the boundary between regions with varying $T_{\mathrm{C}, 0}$.

An example of the magnetisation and local magnetic field, respectively, is given in Fig. 3. The model was set up with the Voronoi maps shown in Fig. 2, an applied field of $0.5 \mathrm{~T}$ and a temperature of $306.5 \mathrm{~K}$. It is clear from the figure that the sample is in a mixed phase 
Table 1. The parameters required by the model and their specific values used in this study. The values are selected to match those of $\mathrm{La}(\mathrm{Fe}, \mathrm{Mn}, \mathrm{Si})_{13} \mathrm{H}$ type materials. The compressibility is taken from Ref. [19]. Please note that the sign of $\beta$, as defined in Eq. 4, is assumed positive.

\begin{tabular}{lll}
\hline Parameter name & Variable & Value used in this work \\
\hline Magneto-elastic coupling & $\eta$ & 1.3 \\
Total angular momentum & $J$ & 1 \\
Curie temperature in the absence of magneto-volume coupling & $T_{\mathrm{C}, 0}$ & $305 \mathrm{~K}$ \\
Mass density & $\rho$ & $7200 \mathrm{~kg} / \mathrm{m}^{3}$ \\
Number of magnetic ions per unit volume & $N / V_{0}$ & $6.04 \times 10^{28} \mathrm{~m}^{-3}$ \\
Number of atoms per unit volume & $N_{\text {atom }} / V_{0}$ & $7.39 \times 10^{28} \mathrm{~m}^{-3}$ \\
Bulk compressibility & $\kappa$ & $7.3 \times 10^{-12} \mathrm{~Pa}^{-1}$ \\
Debye temperature & $T_{\mathrm{D}}$ & $350 \mathrm{~K}$ \\
Sommerfeld constant & $\gamma_{e}$ & $0.24 \mathrm{~J} /(\mathrm{kgK})^{2}$ \\
Pressure & $p$ & $0 \mathrm{~Pa}$ \\
Standard deviation on Curie temperature & $\sigma_{T_{\mathrm{C}, 0}}$ & $0 \mathrm{~K}$ or $2 \mathrm{~K}$ \\
\hline
\end{tabular}

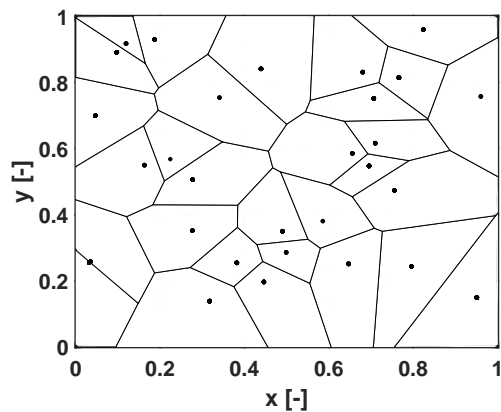

(a)

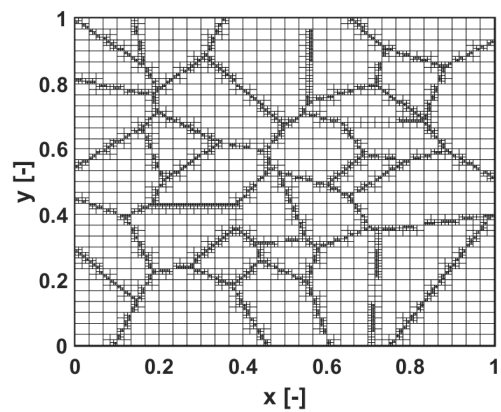

(b)

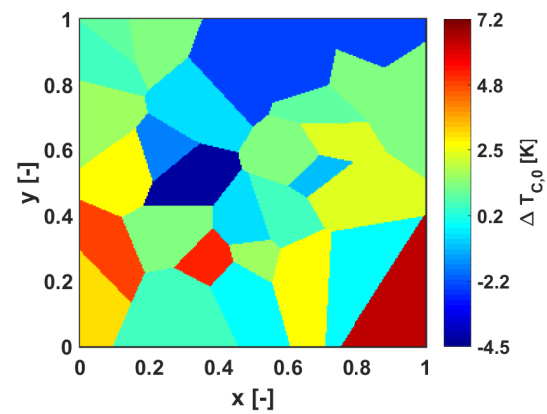

(c)

Figure 2. Example of the generation of the computational domain. In (a) the Voronoi-map is plotted with the locations of the initial 30 random points marked with dots. In (b) the domain has been discretized into a number of rectangular prisms with refinement level $L=4$. The colours in (c) show the difference, $\Delta T_{\mathrm{C}, 0}$ between the average Curie temperature, $T_{\mathrm{C}, 0}$, and the local value.

region, i.e. parts of the sample are para- while others are ferromagnetic. Both the local field and magnetisation are strongly influenced by this effect, which has its origin in the slight variation of Curie temperature $\left(\sigma_{T_{\mathrm{C}, 0}}=2 \mathrm{~K}\right)$.

\subsection{Numerical experiments}

After a specific geometry has been set up and the initial conditions are specified, the model is solved by varying temperature and/or applied magnetic field according to a specified numerical experiment. In the case of an isothermal entropy change experiment the local magnetic field, $\mathbf{H}$, is found at the initial and final states and subsequently the magnetic entropy change is derived.

In the case of an adiabatic temperature change experiment the local field is found at the initial and final states. Then the adiabatic temperature change is found in each prism assuming that the total entropy of each individual prism is conserved during the change in applied field, i.e. the field change is sufficiently fast that any appreciable heat 


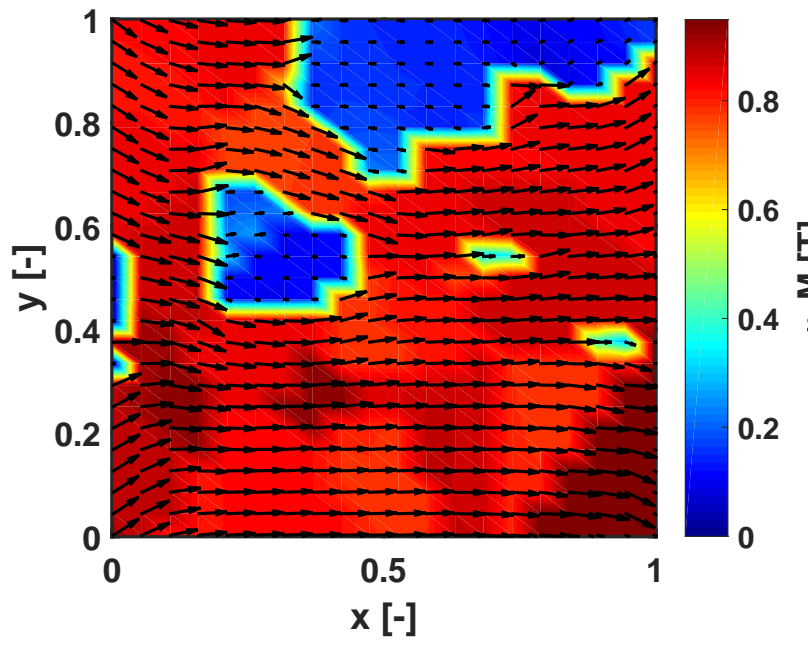

(a)

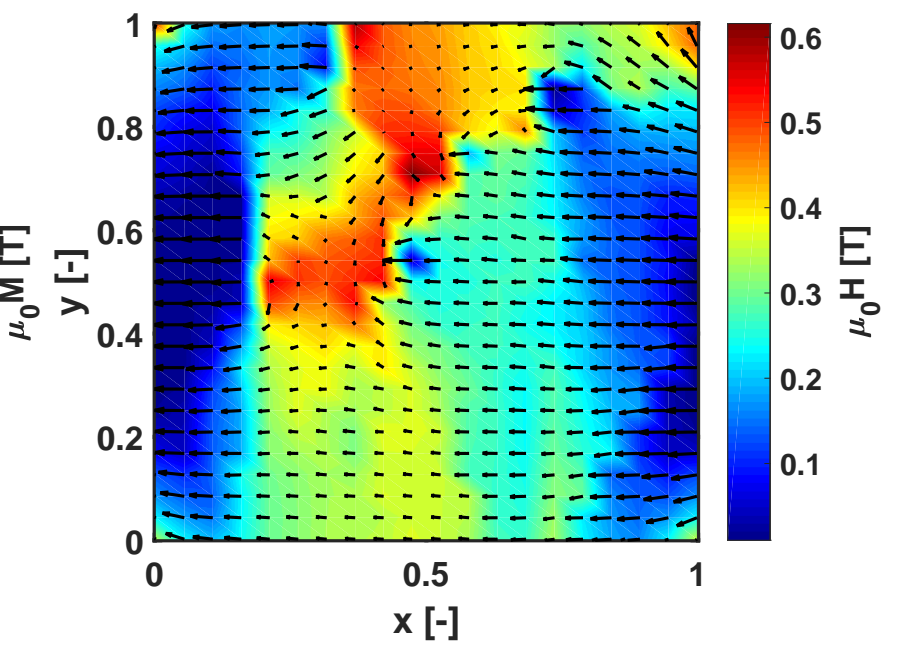

(b)

Figure 3. Contour maps of the magnitude of the magnetisation (a) and the local magnetic field (b), respectively. The arrows indicate the local direction of the magnetisation and magnetic field. The applied field was set to 0.5 $\mathrm{T}$ pointing in the positive $x$-direction, the temperature was $306.5 \mathrm{~K}$ and the Voronoi map is that presented in Fig. 2, i.e. with $\sigma_{T_{\mathrm{C}}}=2 \mathrm{~K}$ and $T_{\mathrm{C}, 0}=305 \mathrm{~K}$. For the field plot the applied field has been subtracted in the arrow plot to emphasise the variation of the field orientation.

transfer does not occur during the field change. It is noted that this assumption is only valid in the limit where the rate of change of the applied field goes to infinity. The system is then relaxed to thermal equilibrium by using the first law of thermodynamics, i.e.

$$
\begin{aligned}
\Delta U=0 & \Rightarrow T_{\mathrm{eq}} \sum_{i=1}^{i=n}\left(\int_{T_{i}}^{T_{\mathrm{eq}}} c_{i}\left(T, H_{i}, h_{i}\right) \mathrm{d} T\right) \\
& =0,
\end{aligned}
$$

with the change in internal energy denoted by $\Delta U$ and the final equilibrium temperature denoted by $T_{\text {eq }}$. The specific heat, temperature, magnetic field norm and state of the $i$ 'th prism are $c_{i}, T_{i}, H_{i}=\sqrt{H_{x, i}^{2}+H_{y, i}^{2}+H_{z, i}^{2}}$ and $h_{i}$, respectively. Equation 15 is solved by numerical minimization on $T \in\left[T_{i}, T_{\text {eq }}\right]$. It is assumed, to first order, that the local field, $\mathbf{H}_{i}$, and local state, $h_{i}$, do not change during the thermal equilibration process. This is an approximation necessary for the current numerical scheme and relies on a sufficiently fast change in the applied magnetic field. The local field and state are, however, allowed to change after the equilibration process and thus iteration is used to obtain the final self-consistent solution. The integral in Eq. 15 is a convenient way to implicity include any latent heat on $\left[T_{i}, T_{\text {eq }}\right]$.

It is noted that the total entropy of each prism must remain constant during the adiabatic magnetisation. However, during the thermal equilibration entropy is not conserved since this is a finite heat transfer process. Even though the total system is isolated each individual prism interacts with all the other prisms from a heat transfer point of view (as well as magnetically) and so an inhomogeneous distribution of properties and/or magnetic field across the sample will lead to a temperature distribution across the sample resulting in entropy generation. 


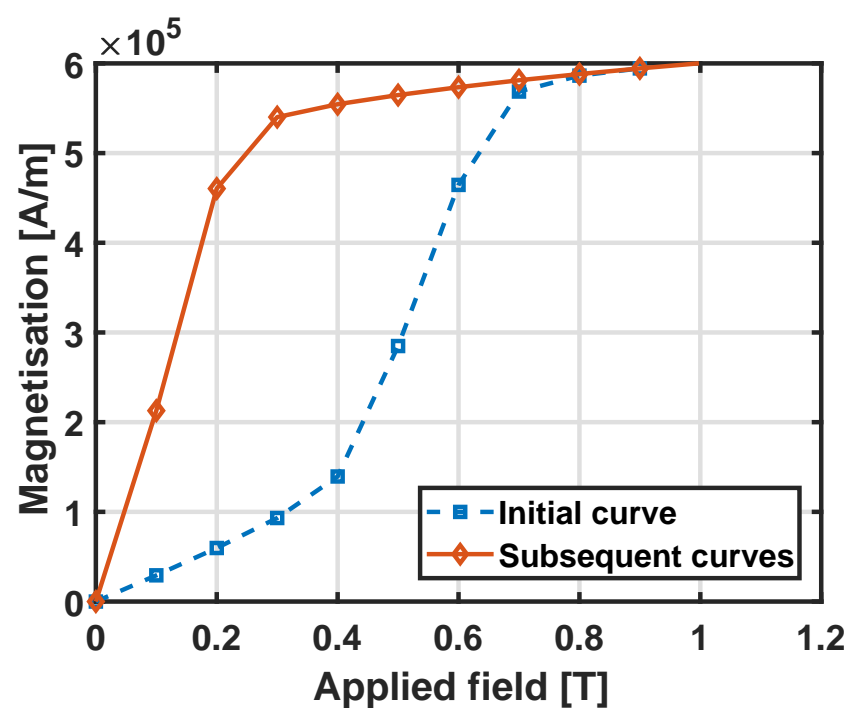

Figure 4. The magnetisation as a function of applied field under isothermal conditions. The blue (dashed) line shows the magnetisation when increasing the first time from a paramagnetically reset state (meta-stable curve). The red (solid) line shows the magnetisation as the field is decreased and increased subsequently (stable curve). The subsequent field loop follows the red line up and down. The variation in Curie temperature was $\sigma_{T_{\mathrm{C}, 0}}=0 \mathrm{~K}$.

\section{Results and discussion}

\subsection{Isotermal entropy change}

In order to model the isothermal entropy change the model was set up for two cases: one with $\sigma_{T_{\mathrm{C}, 0}}=0 \mathrm{~K}$ and one with $\sigma_{T_{\mathrm{C}, 0}}=2 \mathrm{~K}$. The starting temperature of $T=306.5 \mathrm{~K}$ was reached by cooling from an initial fully paramagnetic state. This was chosen to ensure that the sample would be in the mixed phase region, see, e.g., Figure 1. From here the field was cycled from zero to $1.0 \mathrm{~T}$ and back down to zero $\mathrm{T}$ again twice. Figure 4 shows the magnetisation as a function of applied field for these four field changes for the case of $\sigma_{T_{\mathrm{C}, 0}}=0 \mathrm{~K}$. The hysteresis effect is seen to result in two curves: an initial curve and a stable curve.

In the case with a spread in $T_{\mathrm{C}, 0}$ of $2 \mathrm{~K}$ the hysteresis also manifests itself as a minor loop in the magnetisation (Fig. 5(a)). This is thus a direct consequence of the modest variation in $T_{\mathrm{C}, 0}$ across the sample. The influence on the entropy change (Fig. $5(\mathrm{~b})$ ) is quite pronounced; on subsequent magnetisations the entropy change is lowered by a factor of two compared to the value at the first magnetisation.

The phase fraction is seen (Fig. 5(c)) to not reach fully ferromagnetic (a value of one) and the magnetisations/demagnetisations are seen to result in a minor loop similar to that of the magnetisation and entropy change curves.

\subsection{Adiabatic temperature change}

In order to explore the model's ability to handle hysteresis and thermal equilibration an adiabatic field change situation was modelled. A sample with a spread in $T_{\mathrm{C}}$ of $\sigma_{T_{\mathrm{C}, 0}}=$ $2 \mathrm{~K}$ and an initial temperature $T=306.5 \mathrm{~K}$ was considered. The model was assumed to be completely paramagnetic initially, i.e. a thermal reset at high temperature was simulated. The magnetic field was changed from zero to $1.0 \mathrm{~T}$ adiabatically succeeded by thermal equilibration as explained in Section 2.4. Then, subsequently, the applied field was decreased adiabatically from $1.0 \mathrm{~T}$ to zero $\mathrm{T}$ and the sample was once more allowed to equilibrate thermally. Figure 6 shows the resulting adiabatic temperature change as a function of consecutive field changes, i.e. one corresponds to the first application of the field while four corresponds to the second removal of the field, respectively.

A significant initial adiabatic temperature change is seen, which is completely in line with experimental results $[6,21]$. It is important to note that the field change itself, performed under completely adiabatic conditions, cannot 


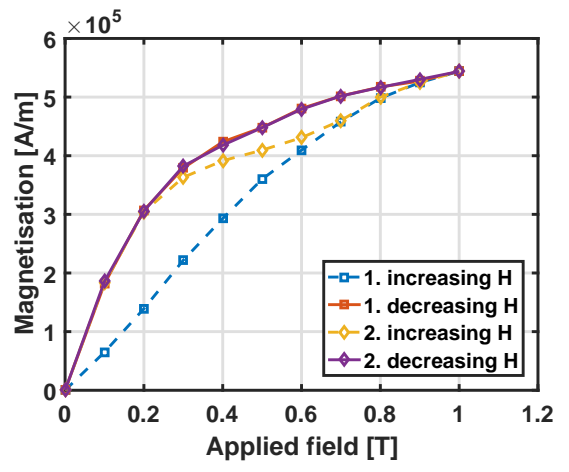

(a)

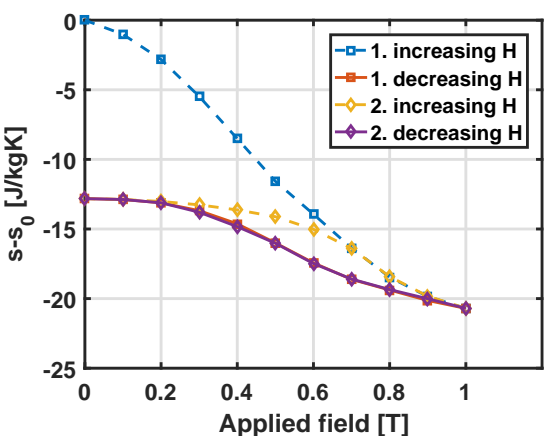

(b)

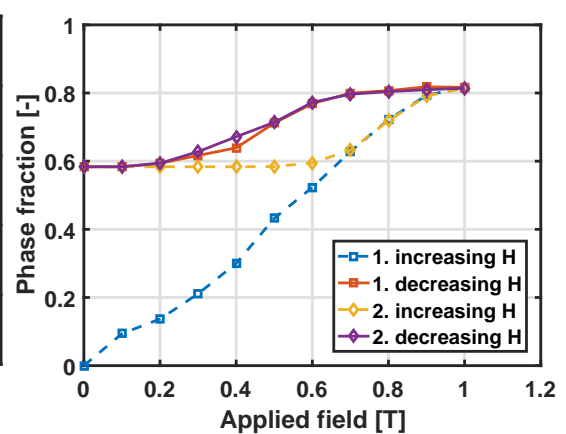

(c)

Figure 5. The magnetisation (a), entropy (b) and phase fraction (c) as functions of applied field under isothermal conditions with $\sigma_{T_{\mathrm{C}, 0}}=2 \mathrm{~K}$.

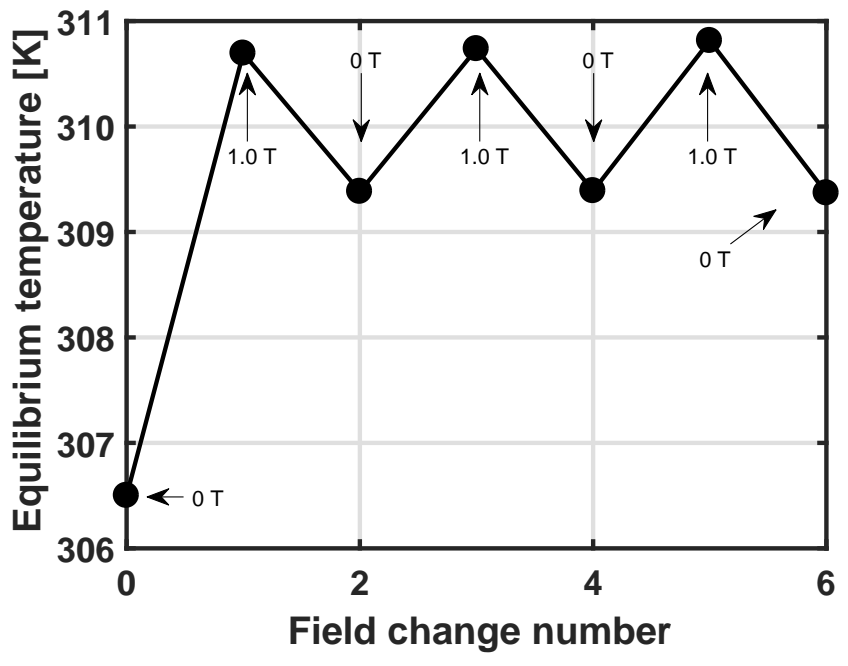

Figure 6. The temperature after adiabatic magnetisation / demagntisation and subsequent thermal equilibration as a function of field change number. The model was initialized in the paramagnetic state and the field was applied/removed three times each. After the first magnetisation the temperature change is seen to be significantly larger than after the subsequent field changes. The annotations indicate the applied field.

give rise to a change in the state of any of the prisms in the model. This is seen by considering the entropy-temperature diagram and keeping in mind that for an adiabatic field change, the total entropy is constant as a function of temperature. However, as the local magnetic field varies due to the demagnetising effects (sample geometry) and the variation in local chemistry (through $\sigma_{T_{\mathrm{C}}}$ ) the prisms will reach different temperatures right after the change in field has occured. This induces heat transfer in the system, which is here modelled through Eq. 15, and this allows the individual prisms to change phase.

In Fig. 7 the phase diagram of the sample is shown in three different cases, i.e. after the first magnetisation $(7(\mathrm{a}))$, after the first demagnetisation $(7(\mathrm{~b}))$ and after the second magnetisation $(7(c))$. Considering the first magnetisation, a significant variation in the resulting temperature and field of the various prisms is observed. Also, a division between ferro- (blue) and paramagnetic (red) prisms in the sample is seen. The reason for this is that the initial temperature, $T_{\text {init }}=306.5 \mathrm{~K}$, was below a significant amount of the prisms' $T_{\mathrm{C}, 0}$ so that they were actually ferromagnetic initially.

After the first equilibration the applied field was removed and a new equilibrium temperature was found (indicated by the black dot in Fig. 7(b)). After the second application of the field, the distribution in the phase diagram (Fig. 7(c)) has changed significantly compared to after the first application of 


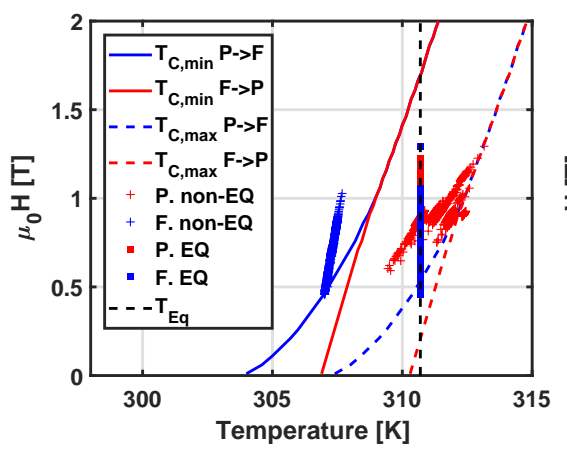

(a)

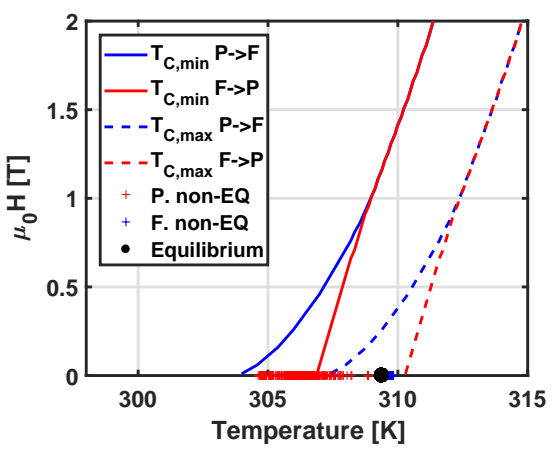

(b)

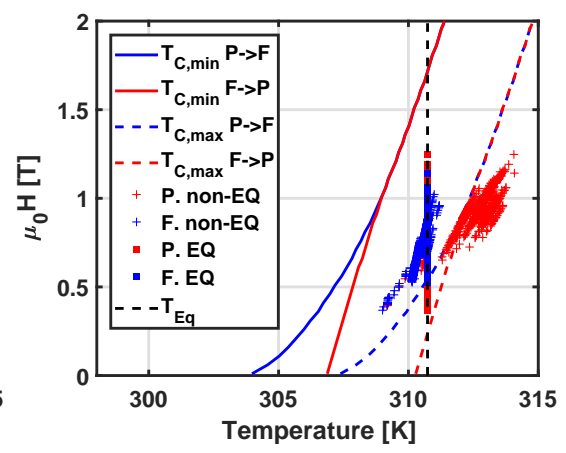

(c)

Figure 7. Distribution of the internal field in a modelled sample magnetising adiabatically. (a) increasing the field from zero to 1.0 initially. (b) lowering the field from 1.0 to zero $\mathrm{T}$ and (c) increasing the field again from zero to $1.0 \mathrm{~T}$. The sample starts with a homogeneous temperature $T_{\mathrm{init}}=306.5 \mathrm{~K}$ and $\sigma_{T_{\mathrm{C}, 0}}=2 \mathrm{~K}$. The solid blue- and red lines indicate the phase lines of the prism with the smallest value of $T_{\mathrm{C}, 0}$ while the dashed blueand red lines indicate the phase diagram for the prism with the highest $T_{\mathrm{C}, 0}$. The black dashed line indicates the final equilibrium temperature. The blue- and red plusses ("+") indicate the ferro- and paramagnetic prisms, respectively, after the magnetisation. Finally, the blue and red squares indicate the ferro- and paramagnetic prisms, respectively, after thermal equlibration.

the field (Fig. 7(a)). However, the final equilibrium temperature is the same in the two cases. Since the equilibrium temperature in the first case of demagnetisation (Fig. $7(\mathrm{~b}))$ is higher than the initial temperature, the resulting adiabatic temperature change is smaller. The reason for this is the fact that heat transfer goes on after the completely adiabatic field change and thus allows local parts of the sample to change phase.

It may be concluded that the initially very large adiabatic temperature change is indeed physical but not reversible. The subsequent lower values of the adiabatic temperature change are due to i) variation throughout the sample due to local magnetic field variations and variation in chemical composition and thus ii) heat transfer, which allows various parts of the sample to change phase during equilibration.

\subsection{First order reversal curves in temperature}

Figure 8 shows reversal curves of the magnetisation as a function of temperature at an applied field of $1.0 \mathrm{~T}$, a value of $\eta=1.5$ and otherwise the same parameters as in Table 1. The higher value of $\eta$ is chosen in order to get a broader hysteresis region. The sample starts at an initial temperature $T_{\text {init }}=310 \mathrm{~K}$ and the temperature is then increased to $T_{1}$ in steps of $0.25 \mathrm{~K}$. Subsequently, the temperature is decreased back to $T_{\text {init }}$ followed by an increase in $T_{1}$ to $T_{1}+1 \mathrm{~K}$ an so on.

In the case of no variation in the Curie temperature (8(a)) some degree of the reversal effect can be seen in the curve that is stopped at $T=314 \mathrm{~K}$. This is caused by the demagnetising field alone. When $\sigma_{T_{\mathrm{C}, 0}}=$ $2 \mathrm{~K}$ clearly the variation in Curie temperature influences the behaviour of the reversal curves as well. Furthermore, the maximum value of the magnetisation is seen to be significantly lowered with a spread (Fig. 8(b) compared to zero variation $8(\mathrm{a})$ ). 


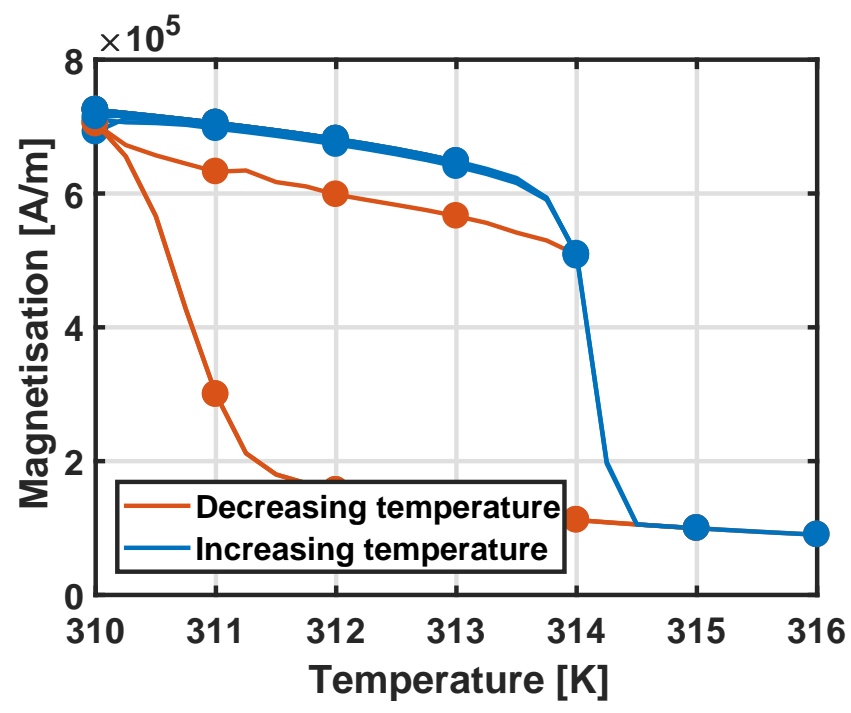

(a)

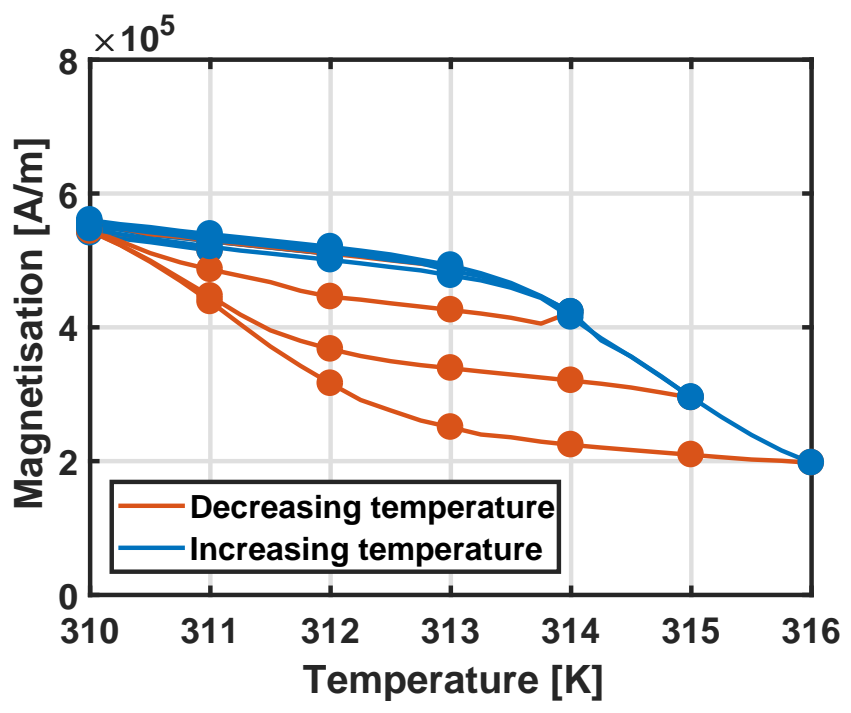

(b)

Figure 8. Magnetisation as a function of temperature at an applied field of $1.0 \mathrm{~T}$. The temperature is ramped to some temperature, $T_{1}$, and then decreased down to the initial temperature, $T_{\text {init }}$. The temperature is then ramped up to $T_{1}+1 \mathrm{~K}$ and cooled down to $T_{\text {init }}$ etc. The spread in $T_{\mathrm{C}, 0}$ is zero in (a) and $\sigma_{T_{\mathrm{C}, 0}}=2 \mathrm{~K}$ in (b). Note that many of the points overlap each other, especially in (a).

The spatial variation in Curie temperature is again seen to influence the overall properties of a given sample in two distinct ways: introducing minor loops and thus a significant history dependence and generally lowering the value of the property under consideration (be it magnetisation, entropy change or adiabatic temperature change). Qualitatively, there is also seen to be a general softening of the properties as functions of either field or temperature when a spatial variation of the Curie temperature is present.

\section{Conclusion and outlook}

A numerical framework for modelling inhomogeneous magnetocaloric materials with a firstorder phase transition was presented and applied to various cases. Through isothermal field changes, adiabatic temperature changes and thermal first order reversal curves we showed that the model captures qualitatively the effects of hysteresis and that even a slight chemical inhomogeneity spatially distributed across a sample results in experimental effects previously reported. These include the thermal first-order reversal curves and virgin isothermal entropy and adiabatic temperature changes.

Assuming zero chemical variation across the sample, we showed that minor loops in the isothermal entropy change versus applied field do not occur. Only when a slight chemical variation was included could these curves be reproduced by the model.

A future version of the model will include finite heat transfer, i.e. temporal resolution of the applied field change. This will enabled direct simulation of various experimental situations such as differential scanning calorimetry.

\section{Acknowledgments}

This work was financed by the ENOVHEAT project which is funded by Innovation Fund 
Denmark (contract no 12-132673).

\section{Appendix A. Demagnetisation tensor field components}

The symmetric $3 \times 3$ demagnetisation tensor field $\mathbb{N}(\mathbf{r}, D)$ has the components (derived in [15]) with the dimensions $D=(a, b, c)$ of the prism considered:

$N_{i i}(\mathbf{r}, D)=$

$\frac{1}{4 \pi}\left(\operatorname{atan} f_{i}(x, y, z, D)+\operatorname{atan} f_{i}(-x, y, z, D)\right.$

$+\operatorname{atan} f_{i}(x,-y, z, D)+\operatorname{atan} f_{i}(x, y,-z, D)$

$+\operatorname{atan} f_{i}(-x,-y, z, D)+\operatorname{atan} f_{i}(x,-y,-z, D)$

$\left.+\operatorname{atan} f_{i}(-x, y,-z, D)+\operatorname{atan} f_{i}(-x,-y,-z, D)\right)$

where

$f_{x}(\mathbf{r}, D)=$

$\frac{(b-y)(c-z)}{(a-x)\left((a-x)^{2}+(b-y)^{2}+(c-z)^{2}\right)^{1 / 2}}($

$f_{y}(\mathbf{r}, D)=$

$\frac{(a-x)(c-z)}{(b-y)\left((a-x)^{2}+(b-y)^{2}+(c-z)^{2}\right)^{1 / 2}}(A$

$f_{z}(\mathbf{r}, D)=$

$\frac{(b-y)(a-x)}{(c-z)\left((a-x)^{2}+(b-y)^{2}+(c-z)^{2}\right)^{1 / 2}}$

The off-diagonal elements are

$N_{i j}(\mathbf{r}, D)=$

$-\frac{1}{4 \pi} \ln \left(\frac{F_{i j}(\mathbf{r}, a, b, c) F_{i j}(\mathbf{r},-a,-b, c)}{F_{i j}(\mathbf{r}, a,-b, c) F_{i j}(\mathbf{r},-a, b, c)}\right.$

$\left.\times \frac{F_{i j}(\mathbf{r}, a,-b,-c) F_{i j}(\mathbf{r},-a, b,-c)}{F_{i j}(\mathbf{r}, a, b,-c) F_{i j}(\mathbf{r},-a,-b,-c)}\right)$

$i \neq j$

where

$F_{x y}(\mathbf{r}, a, b, c)=$

$(c-z)+\left[(a-x)^{2}+(b-y)^{2}+(c-z)^{2}\right]^{1 / 2}$

$F_{y z}(\mathbf{r}, a, b, c)=$

$$
(a-x)+\left[(a-x)^{2}+(b-y)^{2}+(c-z)^{2}\right]^{1 / 2}
$$

$F_{x z}(\mathbf{r}, a, b, c)=$

$(b-y)+\left[(a-x)^{2}+(b-y)^{2}+(c-z)^{2}\right]^{1 / 2}$.

\section{References}

[1] A. Smith, C.R.H. Bahl, R. Bjørk, K. Engelbrecht, K.K. Nielsen, and N. Pryds. Materials challenges for high performance magnetocaloric refrigeration devices. $A d$ vanced Energy Materials, 2:1288-1318, 2012.

[2] A. Kitanovski, J. Tusek, U. Tomc, U. Plaznik, M. Ozbolt, and A Poredos. Magnetocaloric Energy Conversion: From Theory to Applications. Springer International Publishing, New York., 2015.

[3] L. Caron, Z.Q. Ou, T.T. Nguyen, D.T C. Thanh, O. Tegus, and E. Brück. On the determination of the magnetic entropy change in materials with first order transitions. J. Magn. Magn. Mater., 321:3559-3566, 2009.

[4] K.K. Nielsen, H.N. Bez, L. von Moos, R. Bjørk, D. Eriksen, and C.R.H. Bahl. Direct measurements of the magnetic entropy change. Rev. Sci. Instr., 86:103903, 2015.

[5] K. Engelbrecht, K.K. Nielsen, C.R.H. Bahl, C.P. Carroll, and D. van Asten. Material properties and modeling characteristics for $\mathrm{MnFeP}_{1-x} \mathrm{As}_{x}$ materials for application in magnetic refrigeration. $J$. Appl. Phys., 113:173510, 2013.

[6] L. von Moos, C.R.H Bahl, K.K. Nielsen, and K. Engelbrecht. The influence of hysteresis on the determination of the 
magnetocaloric effect in $\mathrm{Gd}_{5} \mathrm{Si}_{2} \mathrm{Ge}_{2} . \quad J$. Phys. D: Appl. Phys., 48:025005, 2015.

[7] V. Franco, T. Gottschall, K.P. Skokov, and O. Gutfleisch. First-Order Reversal Curve (FORC) Analysis of Magnetocaloric Heusler-Type Alloys. IEEE Magnetics Letters, 7:6602904, 2016.

[8] I.D. Mayergoyz. Mathematical models of hysteresis. IEEE Trans. Magn., 22:603, 1986.

[9] V. Basso, C.P. Sasso, and M. LoBue. Thermodynamic aspects of first-order phase transformations with hysteresis in magnetic materials. J. Magn. Magn. Mater, 316:262-268, 2007.

[10] V. Basso, M. Küpferling, C.P. Sasso, and M. LoBue. Modeling hysteresis of first-order magneto-structural phase transformations. IEEE Trans. Magn., 44:3177, 2008.

[11] L. von Moos, C.R.H. Bahl, K.K. Nielsen, K. Engelbrecht, M. Küpferling, and V. Basso. A Preisach approach to modeling partial phase transitions in the first order magnetocaloric material $\mathrm{MnFe}(\mathrm{P}, \mathrm{As}) . \quad$ Physica B, 435:144-147, 2014.

[12] J.D. Moore, K. Morrison, G.K. Perkins, D.L. Schlagel, T.A. Lograsso, K.A. Gschneidner Jr., V.K. Pecharsky, and L.F. Cohen. Metamagnetism Seeded by Nanostructural Features of SingleCrystalline $\mathrm{Gd}_{5} \mathrm{Si}_{2} \mathrm{Ge}_{2}$. Adv. Materials, 21:3780-3783, 2009.

[13] J.M.D. Coey. Magnetism and magnetic materials. Cambridge University Press, New York, 2010.

[14] V. Basso, M. Küpferling, C. Curcio, C. Bennati, A. Barzca, M. Katter, M. Bratko, E. Lovell, J. Turcaud, and
L.F. Cohen. Specific heat and entropy change at the first order phase transition of $\mathrm{La}(\mathrm{Fe}-\mathrm{Mn}-\mathrm{Si})_{13}-\mathrm{H}$ compounds. J. Appl. Phys, 118:053907, 2015.

[15] A. Smith, K. K. Nielsen, D. V. Christensen, C. R. H. Bahl, R. Bjørk, and J. Hattel. The demagnetizing field of non-uniform rectangular prisms. J. Appl. Phys., 107:103910, 2010.

[16] C. P. Bean and D. S. Rodbell. Magnetic disorder as a first-order phase transformation. Phys. Rev., 126:104-115, 1962.

[17] M. Piazzi, C. Bennati, C. Curcio, M. Kuepferling, and V. Basso. Modeling specific heat and entropy change in $\mathrm{La}(\mathrm{FeMnSi})_{13} \mathrm{H}$ compounds. Journal of Magnetism and Magnetic Materials, 400:349-355, 2016. cited By 4.

[18] S. Yahyaoui, M. Khalfaoui, S. Kallel, N. Kallel, J.S. Amaral, and A. Ben Lamine. Modeling the magnetic properties and magnetocaloric effect of $\mathrm{La}_{0.7} \mathrm{Sr}_{0.3} \mathrm{Mn}_{0.9} \mathrm{Ti}_{0.1} \mathrm{O}_{3}$. Journal of Alloys and Compounds, 685:633-638, 2016. cited By 0.

[19] K Irisawa, A Fujita, K Fukamichi, M Yamada, H Mitamura, T Goto, and K Koyama. Transition between antiferromagnetic and ferromagnetic states in itinerant-electron $\mathrm{La}\left(\mathrm{Fe}_{x} \mathrm{Al}_{1-x}\right)_{13}$ compounds. Physical Review B, 70(21):1-16, 2004.

[20] G. Voronoi. Nouvelles applications des paramètres continus à la thèorie des formes quadratiques. Deuxième mèmoire. Recherches sur les parallèlloèdres primitifs. Journal Fur Die Reine Und Angewandte Mathematik, 1908(134), 1908.

[21] J. Liu, T. Gottschall, K.P. Skokov, J.D. Moore, and O. Gutfleisch. Giant magnetocaloric effect driven by structural 
transitions. Nature Materials, 11(7):620626, 2012. 